ISSN: $1130-3743$

\title{
PERCEPCIONES DE LOS DIFERENTES AGENTES IMPLICADOS EN EL SERVICIO DE FORMACIÓN UNIVERSITARIA
}

\author{
Perceptions of different stakeholders involved in Higher \\ Education Service
}

\section{Perceptions des différents agents impliqués dans le Service de Formation Universitaire}

\author{
Teresa Fayos Gardó*, Martina GonZÁlez-Gallarza Granizo* y David SERvera \\ FRANCÉS** \\ * Universidad de Valencia. Facultad de Economía. Departamento de \\ Comercialización e Investigación de Mercados. Av. de los Naranjos, s/n. 46022 \\ Valencia.Correo-e: teresa.fayos@uv.es; martina.gallarza@uv.es \\ ** Universidad Católica de Valencia. Facultad de Estudios de la Empresa. \\ C/ Guillem de Castro, 175. 46003 Valencia. Correo-e: david.servera@ucv.es
}

Fecha de recepción: enero de 2013

Fecha de aceptación definitiva: abril de 2013

Biblid [(1130-3743) 25, 1-2013, 149-172]

RESUMEN

La Universidad en España está en la actualidad en un momento de transformación con la entrada en vigor de los nuevos planes de estudio según los criterios de la declaración de Bolonia de 1999. Teniendo en cuenta la trayectoria de investigación que desde hace unos años se viene observando en las universidades españolas en el estudio de la formación universitaria como un servicio de alta implicación, proponemos un nuevo trabajo en el ámbito de las Titulaciones de Grado en Empresa dentro del nuevo Espacio Europeo de Educación Superior. Concretamente, el trabajo 
se centra en las nuevas competencias que deberían contemplar los nuevos grados de empresa en el ámbito español. Para ello, hemos realizado una serie de consultas para conocer las percepciones de los diferentes agentes implicados en el proceso de formación: alumnos, profesores, egresados, profesionales y empresas. Consultas que nos han permitido obtener información relevante para el desarrollo de los nuevos planes de estudio. sarios.

Palabras clave: Servicio Docencia Universitaria, alumnos, egresados, empre-

\section{SUMMARY}

Universities in Spain are nowadays in a period of transformation due to the adaptation of old degrees to a new structure, proposed in the famous Bologna declaration in 1999. According to a certain research stream that has emerged among service researchers considering education at University as a higher involvement service market, we propose a new work on Business Degrees related to the European Higher Education Area: The study offers a framework where new proposals of Business Degrees in Spain can find information about competences and learning outcomes. We report the results obtained in several queries made to several stakeholders involved in the Higher Education service: students, graduates, teachers and employers (companies). These results are interesting for the strategic development of new degrees.

Key words: Higher Education Service, students, graduates, employers.

\section{SOMMAIRE}

L'université espagnole se trouve actuellement dans un moment de fortes transformations avec l'entrée en vigueur des nouveaux diplômes en accord avec les critères que propose la déclaration de Bologne de 1999. Compte tenu de l'existence d'un courant de recherche qui s'est dernièrement développé dans les universités espagnoles autour de l'analyse du Service de Formation Universitaire comme un service de haute implication, nous proposons un nouveau travail sur les diplômes de commerce et management dans le cadre de l'Espace Européen de l'Enseignement Supérieur. D’une manière plus précise, ce travail est une contribution a l'analyse des nouvelles compétences que les étudiants de business en Espagne devraient développer dans ces nouveaux diplômes (Grados), créé a partir d'une série de consultations effectuées auprès des différents agents impliqués (stakeholders) dans le processus de formation, á savoir: les étudiants, les professeurs, les diplômés, les entreprises et les employeurs. L'information recueillie est d'utilité pour le suivi stratégique de la mise en place et du développement de ces nouveaux diplômes.

Mots clés: Service de Formation Universitaire, etudiants, diplômés, employeurs. 
1. Marco teórico: el Servicio de Formación Universitaria en SU PResente Y FUTURO

\subsection{La implantación el Proceso de Bolonia en España}

La adaptación de la Universidad española al Espacio Europeo de Educación Superior y, por tanto, la adopción de métodos comunes en las universidades europeas, ha supuesto cambios importantes: implantación de un sistema de educación superior con dos ciclos, establecimiento de un sistema de acreditaciones basado en el volumen de trabajo dedicado por el alumno y fomento de la calidad educativa en el ámbito universitario (Università degli Studi di Bologna, 1999; Ley Orgánica 4/2007; R. D. 1044/2003; R. D. 1467/2007; R. D. 1125/2003; R. D. 1993/2007; Consejo... Economistas, 2008; De Miguel et al., 2005; Borrador ANECA, 2007; Zabalza, 2004; Manzaneque y Barba, 2008). Nos enfrentamos pues a un cambio estructural y de enfoque que ha de acompañarse necesariamente de cambios en la metodología de enseñanza-aprendizaje y en la evaluación de aprendizajes, que fomenten la formación de los estudiantes no sólo en conocimientos, sino también en competencias (Consejo de Coordinación Universitaria del MEC, 2003, 27).

Como institución al servicio de la sociedad, la Universidad del siglo XXI entiende que, a su misión de formar y generar conocimiento a través de la investigación, se suma cada vez más la de contribuir de manera activa a la generación de riqueza, desarrollando su labor formativa e investigadora de una forma más comprometida con las necesidades de la sociedad (Juliá, 2008). Adicionalmente, las instituciones de la UE (Consejo Europeo, Instrucción y formación 2010: la urgencia de las reformas para el impulso de la estrategia de Lisboa, 3-3-2004. Documento del Consejo 6905/2004) han subrayado el carácter no sólo económico sino también social de los sistemas de formación. La educación superior es por tanto un factor relevante para incrementar las oportunidades de los individuos, favoreciendo su participación en la vida laboral y social y por esta vía aumentando su bienestar social (Ceular et al., 2008).

Desde esta reflexión, y siguiendo a Juliá (2008), coincidimos en señalar que Bolonia supuso un acuerdo de los diferentes sistemas universitarios europeos sobre la conveniencia de converger hacia un sistema más armonizado pero, sobre todo, más eficiente. Se trataba de facilitar una mayor respuesta a la demanda de perfiles profesionales que a su vez favoreciera una más rápida inserción laboral, aportando capital humano y conocimiento en nuestras empresas, y también de facilitar el intercambio y la movilidad de alumnos y profesores de dichos sistemas. El mercado sería así una referencia obligatoria que, sin que sea excluyente, debe ser al menos observada y atendida. Eso significa que esa demanda debe atender, por un lado, al mercado de trabajo y, por otro, 


\section{TERESA FAYOS GARDÓ, MARTINA GONZÁLEZ-GALLARZA GRANIZO Y DAVID SERVERA FRANCÉS}

PERCEPCIONES DE LOS DIFERENTES AGENTES IMPLICADOS EN EL SERVICIO DE FORMACIÓN UNIVERSITARIA

a los deseos de cursar determinados perfiles profesionales por parte de los jóvenes que ingresan en las universidades (Juliá, 2008) ${ }^{1}$.

No obstante, el debate permanece abierto y nuevos interrogantes deberían ser tenidos en consideración. En este sentido cabe plantearse el riesgo de centrar la formación en la consecución de un empleo a corto plazo (primer empleo) olvidando determinadas parcelas formativas que serían útiles para la consecución de empleos a más largo plazo y que implicasen mayor responsabilidad y diferentes destrezas. En este sentido existe amplia literatura sobre el acceso de los universitarios al primer empleo (Rahona, 2004, 2006; Vidal García, 2003; Corrales y Rodríguez, 2003; Burke, 1997) y empiezan a aparecer investigaciones que plantean otro tipo de relaciones no tan a corto plazo, como por ejemplo las de Ceular et al. (2008), que diferencian entre el acceso al primer empleo y el empleo "más estable". El escenario actual de crisis con altas cifras de paro debe necesariamente hacernos reflexionar sobre este aspecto.

Como es sabido, el curso 2008-2009 fue el primer curso de implantación de este tipo de enseñanzas en España. Tras un proceso de verificación, de acuerdo con los datos del Informe de la ANECA al Consejo de Universidades (2008), el número de títulos con informe favorable alcanzó la cifra de 163, lo que representa un $81 \%$ de las presentadas inicialmente al proceso de verificación ( $c f$. Primera columna del Cuadro 1).

CUAdro 1. SOlicitud DE Nuevos GRAdOS EN ESPAÑA (2008 y 2009)

\begin{tabular}{|l|c|c|c|c|c|c|}
\hline \multicolumn{1}{|c|}{ RAMA DE CONOCIMIENTO } & \multicolumn{2}{|c|}{ FAVORABLES } & \multicolumn{2}{c|}{ DESFAVORABLES } & \multicolumn{2}{c|}{ PRESENTADOS } \\
\hline & 2008 & 2009 & 2008 & 2009 & 2008 & 2009 \\
\hline Artes y Humanidades & $26(87 \%)$ & $242(99 \%)$ & $4(13 \%)$ & $3(1 \%)$ & 30 & 245 \\
\hline Ciencias & $9(82 \%)$ & $143(99 \%)$ & $2(18 \%)$ & $1(1 \%)$ & 11 & 144 \\
\hline Ciencias Sociales y Jurídicas & $73(87 \%)$ & $475(96 \%)$ & $11(13 \%)$ & $21(4 \%)$ & 84 & 496 \\
\hline Ingeniería y Arquitectura & $30(73 \%)$ & $215(94 \%)$ & $11(27 \%)$ & $13(6 \%)$ & 41 & 228 \\
\hline Ciencias de la Salud & $25(74 \%)$ & $172(98 \%)$ & $9(26 \%)$ & $3(2 \%)$ & 34 & 175 \\
\hline Presentados & $163(82 \%)$ & $1.247(97 \%)$ & $37(18 \%)$ & $41(3 \%)$ & 200 & 1.288 \\
\hline
\end{tabular}

Fuente: Informe 2009 sobre el estado de la evaluación externa de la calidad en las universidades españolas (ANECA).

A pesar del elevado número de informes favorables, es necesario constatar que fue muy escasa la solicitud de verificación, y en particular fueron muy pocas las universidades públicas que iniciaron el proceso en su primera edición. De

1. Juan Juliá, rector de la Politécnica de Valencia, Suplemento de El Mundo Campus, 30 de abril de 2008 . 
los 2.600 títulos existentes en España en 2007-2008 sólo 200 (7,69\%) siguieron completo el proceso de acreditación para poder impartir las enseñanzas de grado el siguiente curso. Según estos datos del primer año se cumplió, para el caso de España, la proposición de Neave y Amaral (2008) cuestionando la idoneidad de la estrategia asumida cara a completar en el 2010 tal y como estaba prevista la nueva estructura de grados y sus instrumentos. El Cuadro 1 recoge comparativamente las cifras del año 2009, en donde se aprecia un incremento del más del 600\% en los títulos presentados a evaluación. Así, es sólo ese segundo año el que ha asegurado una verdadera recuperación en el proceso permitiendo augurar un cumplimiento de los plazos establecidos.

Con las cifras en la mano, un apunte más que reseñable es que, en el 2008, el 64\% de estas solicitudes de homologación pertenecen a universidades privadas y de la Iglesia católica -más concretamente un 51\% a la primera y un 13\% a la segunda-. Esto implica que sólo un 36\% de las peticiones pertenecen a instituciones públicas (El Mundo, Campus, 20 de febrero 2008).

Ante este escenario temporal y espacial, el presente trabajo quisiera aportar a la comunidad científica interesada en el estudio de la formación universitaria un análisis de este servicio y de su consumo desde una perspectiva de marketing, que además pueda ayudar a comprender mejor la necesidad de realizar un diseño estratégico de las nuevas titulaciones de Grado en Empresa para el caso particular de España. Para ello revisamos a continuación la investigación académica anterior sobre las evaluaciones de los estudiantes universitarios en su escenario de consumo, para proponer después los objetivos de nuestra investigación.

\subsection{El estudiante universitario como sujeto consumidor: el corazón de un complejo entramado de relaciones}

De la revisión efectuada sobre la investigación de las diferentes variables anteriores y posteriores a la elección de un centro universitario ( $c f$. Cuadro 2), apuntamos en un primer momento como las tres estrategias básicas de marketing han sido estudiadas por los autores interesados en el servicio universitario: segmentación (e. g. Oosterbeck et al., 2002; Fuentes y Gil, 2006; Fuentes, Gil y Berenguer, 2005), diferenciación (e. g. Parameswaran y Glowacka, 1995; Luque y Del Barrio, 2007) y posicionamiento (e. g. Comm y Labay, 1996; Ivy, 2001; Maringe, 2006). Esta realidad es un reflejo de que la concepción estratégica de este servicio, público o privado, es cada vez más necesaria.

De manera más pormenorizada, del estudio de la literatura que aparece reflejado en el Cuadro 2, en la etapa anterior a la compra y en el momento de la selección se manifiestan como aspectos más importantes las circunstancias socioeconómicas, mientras que en el comportamiento en la etapa postcompra el interés ha estado tradicionalmente centrado en la imagen y la calidad del servicio (e. g. Osterbeck et al., 1992; Fuentes, Gil y Berenguer, 2005), pudiéndose apreciar sin embargo recientemente un creciente interés en el valor y la lealtad (e. g. Leblanc 


\section{TERESA FAYOS GARDÓ, MARTINA GONZÁLEZ-GALLARZA GRANIZO Y DAVID SERVERA FRANCÉS}

PERCEPCIONES DE LOS DIFERENTES AGENTES IMPLICADOS EN EL SERVICIO DE FORMACIÓN UNIVERSITARIA

y Neuguyen, 1999; Nguyen y Leblanc, 2001). Todas estas son variables importantes para la correcta comprensión de las evaluaciones del Servicio de Formación Universitaria. En ese sentido, recientemente, algunos autores (Filippakou y Ttapper, 2008; Ribera-Camino y De Juan, 2007; Bekkering y Engelland, 2002) insisten en la necesidad de seguir trabajando desde las universidades en la línea del incremento y aseguramiento de la calidad.

Dentro de la investigación de este consumo, desde un punto de vista de gestión de la oferta universitaria, conviene subrayar la consideración de la universidad como un mercado y sus servicios como un producto, en donde son destacables: la búsqueda de la satisfacción del estudiante como un objetivo adicional, la realización de promociones más sofisticadas o reinventar la promoción (Fielder et al., 2003; Hemsley-Brown, 2006; Krampf y Heinlein, 1981: Vander Schee, 2010; Pampaloni, 2010), el creciente interés de los empleadores por la imagen de la universidad, y la aparición de nuevas categorías de profesionales en las universidades, lo que refleja cambios en las necesidades institucionales de las propias universidades. En esta línea, Whitchurch (2008) ha identificado la aparición de una nueva categoría de responsables de las instituciones universitarias que mezclan elementos profesionales y académicos, mientras que Smith y Adams (2008) apuntan que los pro-vice-chancellors de las universidades británicas se han visto obligados a adoptar estilos de dirección ejecutiva para el liderazgo y toma de decisiones.

De lo expuesto hasta el momento, a pesar de alguna reserva como la de Svenson y Wood (2007) no hay duda de la importancia del estudiante como sujeto consumidor del producto-servicio universitario. No obstante, la riqueza de este escenario de consumo estriba en que el estudiante será posteriormente a su vez considerado un sujeto pasivo que es "consumido" por el empresario, por lo que la bondad del producto se constatará posteriormente por su empleabilidad, una de las condiciones que apunta Bolonia; por ello, no sólo es necesario conocer las preferencias y deseos de los estudiantes, sino también, las expectativas de los profesionales como un stakeholder importante evaluador del "producto" final de la formación universitaria, y complementariamente las percepciones de los recientes egresados, ya incorporados al mercado de trabajo y anteriormente estudiantes, por lo que disponen de una más rica percepción.

Si a esta duplicidad de sujetos consumidores le sumamos la importancia del prestador del servicio o elemento de personal en contacto, los profesores, debemos empezar a comprender el servicio universitario como multidimensional. La multiplicidad de agentes que intervienen en el servicio universitario es así cada vez más amplia, debiendo todos los colectivos necesariamente ser tenidos en cuenta desde un punto de vista estratégico a la hora de diseñar productos y servicios destinados al consumo de los estudiantes. 
TERESA FAYOS GARDÓ, MARTINA GONZÁLEZ-GALLARZA GRANIZO Y DAVID SERVERA FRANCÉS

CuAdro 2

REVISIÓN BIBLIOGRÁficA DE LA EVOlUCIÓN DEL SERVICIO DE FORMACIÓN UNIVERSITARIA
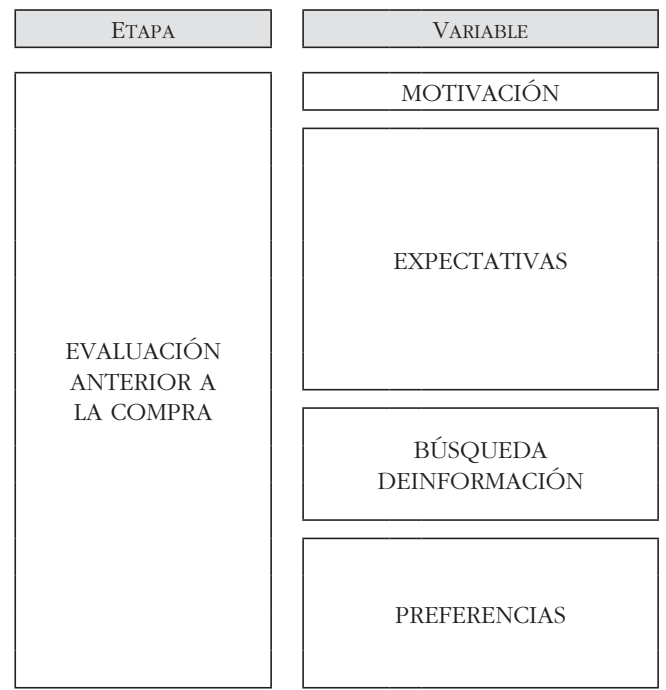

\begin{tabular}{|c|c|c|}
\hline AuTORES \\
\hline Cuestas, Fenollar y Román (2006)
\end{tabular}

Osterbeek, Groot y Hartog (1992)

Belanger, Mount y Wilson (2002)

Fuentes, Gil y Berenguer (2005)

Willis (2005)

Appleton-Knapp y Krentler (2006)

Fuentes y Gil (2006)

Fuentes, Gil y Moliner (2007)

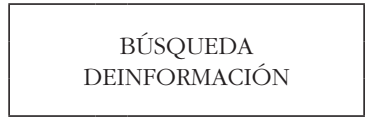

Veloutsou, Lewis y Paton (2004)

Angulo, Pergelova y Rialp (2010)

Pampaloni (2010)

Osterbeek, Groot y Hartog (1992)

Soutar y Turner (2002)

PREFERENCIAS

Holsworth y Nind (2005)

Angulo, Pergelova y Rialp (2010)
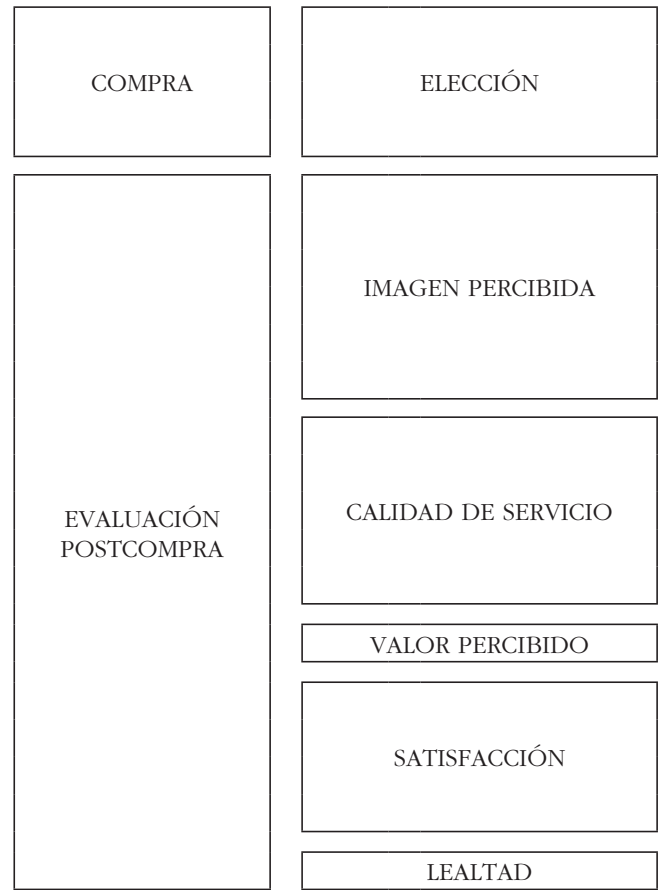

Boone, Olffen y Roijakkers (2004)

Sbanka, Quintal y Taylor (2005)

Mansfield y Warwick (2005)

Maringe (2006)

Osterbeek, Groot y Hartog (1992)

Grady, Fisher y Fraser (1996)

Leblanc y Nguyen (1999)

Ivy (2001)

Nguyen y Leblanc (2001)

Beerli y Díaz (2003)

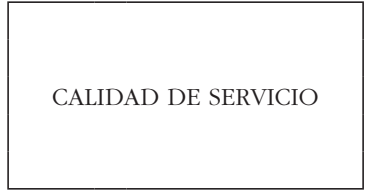

Enegelland, Workman y Sing (2000)

Fuentes, Gil y Berenguer (2005)

Fuentes y Gil (2006)

Fuentes, Gil y Moliner (2007)

Athiyaman (2007)

VALOR PERCIBIDO

Leblanc y Nguyen (1999)

Beerli y Díaz (2003)

Arambewela, Hall y Zuhair (2005)

Appleton-Knapp y Krentler (2006)

Athiyaman (2007)

Nguyen y Leblanc (2001) 
156 TERESA FAYOS GARDÓ, MARTINA GONZÁLEZ-GALLARZA GRANIZO Y DAVID SERVERA FRANCÉS PERCEPCIONES DE LOS DIFERENTES AGENTES IMPLICADOS EN EL SERVICIO DE FORMACIÓN UNIVERSITARIA

2. PlanteAmiento DE LA INVESTigación

\subsection{Objetivos del trabajo}

Del marco teórico expuesto más arriba así como de la revisión bibliográfica efectuada se desprenden una serie de escenarios de investigación; en la presente propuesta pretendemos analizar de manera exploratoria alguno de ellos.

Estos escenarios de investigación buscan arrojar luz sobre la particularidad y complejidad del entorno de consumo del Servicio de Formación Universitaria. Entendiéndolos mejor, podremos realizar un análisis descriptivo que ilustre la particularidad del Servicio de Formación Universitaria.

De manera más concreta, para este trabajo, son de interés dos de ellos: el de la multidimensionalidad del Servicio de Formación Universitaria y el de la multiplicidad de agentes implicados en el encuentro de servicio universitario. A ellos sumaremos un tercer escenario más contextualizado en el momento actual de adaptación al Espacio Europeo de Educación Superior en España como es el del interés por la empleabilidad como resultado de la formación universitaria (Tchibozo, 2007; Núñez, 2007a, 2007b).

Estos tres escenarios darán lugar a tres objetivos de investigación que a su vez se instrumentalizarán en diferentes cuestiones a investigar como a continuación planteamos.

El primer escenario apuntado da lugar al primero de los objetivos de este trabajo, que se entiende como de continuidad respecto al trabajo de Gallarza et al. (2008): se trata de dar un soporte empírico a la idea de multidimensionalidad del Servicio de Formación Universitaria. Los autores en ese trabajo anterior demostraron empíricamente que los llamados paraservicios adquirían desde la perspectiva del consumidor (estudiante) la misma importancia que el servicio académico en sí. Por tanto, es necesario investigar si en el nuevo escenario en el que nos encontramos los servicios relacionados pero no estrictamente académicos continúan siendo tan importantes como los académicos.

En lo relativo al segundo escenario, la multiplicidad de agentes implicados, tomamos como punto de partida el análisis efectuado en Servera et al. (2008), en el que se propone, como conclusión derivada del análisis teórico efectuado sobre las particularidades del Servicio de Formación Universitaria, que "podemos destacar de su análisis la multiplicidad de agentes implicados en el encuentro de servicio (profesores, personal de administración y servicios, padres, estudiantes, responsables académicos...". Para un correcto diseño del producto-servicio universitario bajo el soporte de unas nuevas titulaciones se debe ampliar el prisma de análisis desde los estudiantes hasta otros agentes implicados o stakeholders. La investigación a realizar deberá por tanto incluir las valoraciones del máximo número de dichos agentes: estudiantes, profesores, egresados, etc.

En tercer lugar, y con el deseo de contextualizar esta investigación en el momento actual que vive la Universidad española, proponemos un tercer escenario de investigación de este trabajo relativo a uno de los postulados máximos de la 
Declaración de Bolonia que exponíamos anteriormente, a saber, la búsqueda de la empleabilidad.

A este respecto indudablemente, dentro de los objetivos perseguidos por los estudiantes como consumidores habría que destacar lo referente a sus expectativas profesionales. Estas han sido un objetivo de muchos de los trabajos de la línea de investigación sobre comportamiento del consumidor en el servicio de formación (e. g. Holsworth y Nind, 2005; Soutar y Turner, 2002; Maringe, 2006). Así, se ha destacado el interés del alumno por el paso al mercado laboral, no sólo como indicador de sus preferencias a la hora de elegir carrera y centro (comportamiento anterior a la compra), sino también como uno de los elementos más valorados por los estudiantes en la evaluación del servicio recibido en algunos estudios (comportamiento posterior a la compra). Esta realidad obliga a las universidades a diferenciarse por el alto grado de empleabilidad, lo que en realidad ya venía apuntándose como prioritario en la declaración de Bolonia de 1999. Este escenario de investigación nos ofrece el tercer objetivo propuesto por este trabajo: pulsar y explorar entre los diferentes agentes implicados en la formación universitaria el interés por la empleabilidad que de manera teórica propone el proceso de Bolonia. Esta información debería resultar de gran utilidad para el diseño de nuevos Planes de Estudio en el marco del EEES, es decir, para la propuesta y lanzamiento al mercado de "nuevos productos" atractivos para el consumidor y diferenciados de la competencia.

\subsection{Colectivos implicados y cuestiones a investigar}

Del marco teórico expuesto hasta el momento así como de la revisión bibliográfica planteamos las siguientes cuestiones a investigar para alcanzar los objetivos de este trabajo. Cuatro son los públicos que hemos elegido para nuestro estudio como importantes stakeholders del servicio de formación: los estudiantes (divididos a su vez en estudiantes actuales, delegados y los implicados en las consultas específicas), los egresados, los empleadores y los profesores. Sobre cada uno de ellos planteamos unas cuestiones a investigar.

Respecto a las preferencias de los estudiantes, sería interesante conocer si los estudiantes otorgan mayor importancia a los servicios académicos que a los paraservicios, cuál es el nivel de satisfacción con unos y otros servicios, y cuáles son las asignaturas a las que asignan un mayor grado de importancia en su formación.

La opinión de los delegados consideramos que es interesante ya que representan al grupo líder y más implicado del colectivo de estudiantes, siendo al tiempo el grupo más en contacto con el profesorado y la dirección de la universidad, por lo que interesan especialmente sus propuestas cara a la mejora de las relaciones autoridades académicas-alumnado.

Respecto a las preferencias de los empleadores, es decir, las empresas, es necesario investigar el grado de importancia que asignan a las distintas habilidades y conocimientos de los alumnos a la hora de su inserción en la empresa; 
158 TERESA FAYOS GARDÓ, MARTINA GONZÁLEZ-GALLARZA GRANIZO Y DAVID SERVERA FRANCÉS

PERCEPCIONES DE LOS DIFERENTES AGENTES IMPLICADOS EN EL SERVICIO DE FORMACIÓN UNIVERSITARIA

esta información resultará útil para un diseño de servicio basado en competencias como es el de la Convergencia Europea.

La opinión de los egresados sobre la utilidad de los distintos conocimientos y habilidades aprendidos durante sus estudios una vez insertados en el mundo empresarial sería otra de las cuestiones a investigar que complementaría, por una parte, sus opiniones como estudiantes anteriores a su inserción y, por otra, complementa la de los empleadores facilitando el objetivo de la empleabilidad.

Respecto a los profesores, las cuestiones a investigar serían su opinión sobre el programa formativo, sobre la organización de la enseñanza y sobre el proceso de enseñanza-aprendizaje, como elementos endémicos y vitales que son de todos estos aspectos.

\section{Metodología}

Para la consecución de los objetivos planteados, y con carácter exploratorio, se realizó una investigación cuantitativa, a través del pase de cuestionarios estructurados (previamente pretestados) a diferentes colectivos con diferentes porcentajes de respuesta en función de las poblaciones, obteniendo así diferentes muestras de conveniencia representativas de los diferentes colectivos implicados en la prestación del servicio universitario. Este trabajo de campo se realiza en dos titulaciones de distinto nivel de una misma facultad: la licenciatura en ADE (Administración y Dirección de Empresas, titulación de 5 años) y la diplomatura de Empresariales (CC. EE.). Dichos cuestionarios se complementaron con información secundaria ya disponible de encuestas que se realizan periódicamente o puntualmente en la universidad. En la siguiente figura (Figura 1) se recogen todos los colectivos consultados y las materias sobre las que se les consultó, mientras que en el Cuadro 3 se recogen los datos relativos a la población, porcentaje de respuesta, muestra y características de la misma.

Dado que la investigación que se presenta en este trabajo es de carácter exploratorio los resultados aportados serán de carácter descriptivo: medias y desviaciones típicas de las distintas variables, todas ellas medidas con escalas Likert de 5 puntos (excepto para el cuestionario de los delegados que se utilizó una escala Likert de 4 puntos). Por otra parte, dado el elevado número de resultados obtenidos, se presentan tanto tablas de resultados de la mayoría de los colectivos considerados, como apreciaciones y comentarios sobre todos los colectivos estudiados. 


\section{Figura 1. COlECTIVOS CONSUltados Y MATERIAS DE CONSUlTA}

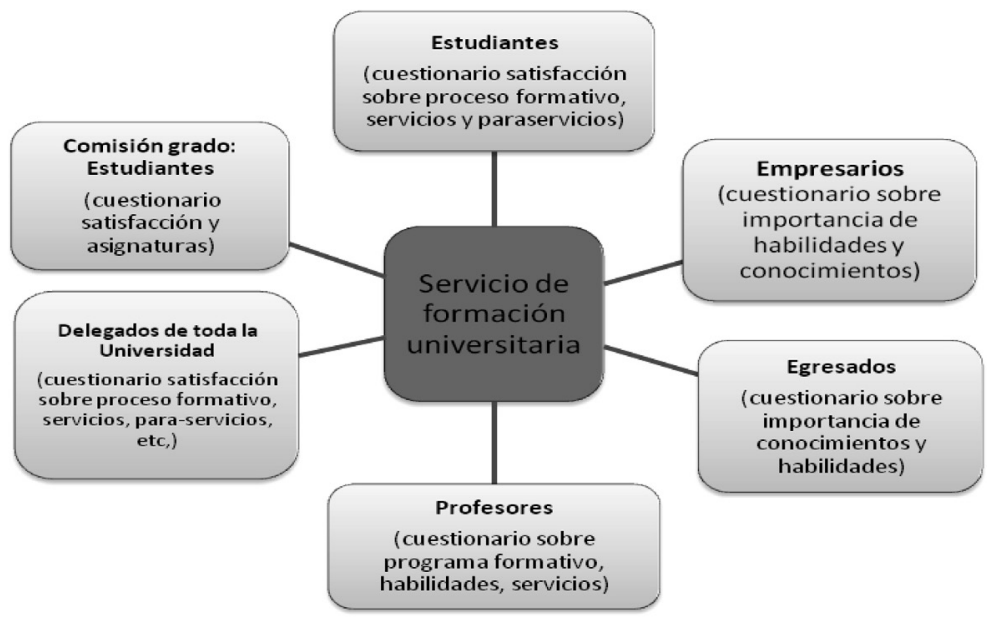

CUADro 3. COlEctivos CONSUltados

\begin{tabular}{|c|c|c|c|c|}
\hline COLECTIVO & POBLACIÓN & $\begin{array}{c}\% \\
\text { RESPUESTA } \\
\end{array}$ & MuestRA & CARACTERÍSTICAS \\
\hline Estudiantes & $\begin{array}{c}245 \text { (ADE) } \\
205 \text { (CC. EE.) }\end{array}$ & $\begin{array}{l}25,3 \% \\
26,8 \%\end{array}$ & $\begin{array}{c}62 \text { (ADE) } \\
55 \text { (CC. EE.) }\end{array}$ & $\begin{array}{l}\text { Representan hombres/mujeres en porcentajes } \\
\text { similares, lo que es consecuente con la } \\
\text { distribución de género del alumnado. }\end{array}$ \\
\hline $\begin{array}{l}\text { Comisión } \\
\text { de grado: } \\
\text { estudiantes }\end{array}$ & $\begin{array}{c}245 \text { (ADE) } \\
205 \text { (CC. EE.) }\end{array}$ & $\begin{array}{l}20,0 \% \\
11,7 \%\end{array}$ & $\begin{array}{l}49 \text { (ADE) } \\
24 \text { (CC. EE.) } \\
72(*)\end{array}$ & $\begin{array}{l}\text { El } 82 \% \text { de los alumnos son estudiantes a } \\
\text { tiempo completo aunque hay alumnos } \\
\text { que trabajan por cuenta ajena (11\%) o } \\
\text { trabajadores autónomos }(4 \%)\end{array}$ \\
\hline $\begin{array}{l}\text { Delegados } \\
\text { de toda la } \\
\text { universidad }\end{array}$ & 74 & $100,0 \%$ & 74 & $\begin{array}{l}\text { Representan hombres/mujeres en porcentajes } \\
\text { similares, lo que es consecuente con la } \\
\text { distribución de género del alumnado. }\end{array}$ \\
\hline $\begin{array}{l}\text { Egresados } \\
\text { curso 2006- } \\
2007 \\
\end{array}$ & $\begin{array}{c}55 \text { (ADE) } \\
35 \text { (CC. EE.) }\end{array}$ & $\begin{array}{l}15 \% \\
23 \%\end{array}$ & $\begin{array}{c}8 \text { (ADE) } \\
8 \text { (CC. EE.) }\end{array}$ & $\begin{array}{l}\text { Representan hombres/mujeres en porcentajes } \\
\text { similares. }\end{array}$ \\
\hline $\begin{array}{l}\text { Empresarios } \\
\text { (prácticas de } \\
\text { empresa ADE } \\
\text { y CC. EE.) }\end{array}$ & 141 & $34,0 \%$ & 48 & $\begin{array}{l}\text { Representan a empresas grandes (16), medianas } \\
\text { (14) y pequeñas (18) casi por igual. } \\
\text { El 34\% de los encuestados tiene un nivel de } \\
\text { estudios "Doctor o Máster" seguidamente de } \\
\text { "licenciados" (29\%) y Diplomados (24\%). } \\
\text { La edad media es de 38,7 años. }\end{array}$ \\
\hline Profesores & 22 & $100,0 \%$ & $\begin{array}{l}16(\mathrm{ADE})^{* * *} \\
9(\mathrm{CC} . \mathrm{EE} .)^{* * *}\end{array}$ & $\begin{array}{l}\text { Mayoría de mujeres lo que es consecuente con } \\
\text { la distribución de género del profesorado. }\end{array}$ \\
\hline
\end{tabular}

(*) Uno de los alumnos estaba matriculado en ambas titulaciones y únicamente respondió a un cuestionario sobre los aspectos generales del centro.

(**) Algunos profesores docentes en ambas titulaciones respondieron a ambos cuestionarios. 
160 TERESA FAYOS GARDÓ, MARTINA GONZÁLEZ-GALLARZA GRANIZO Y DAVID SERVERA FRANCÉS PERCEPCIONES DE LOS DIFERENTES AGENTES IMPLICADOS EN EL SERVICIO DE FORMACIÓN UNIVERSITARIA

\section{Resultados}

\subsection{La perspectiva del alumnado}

De las encuestas realizadas a los alumnos, se desprende que pese a que el nivel de satisfacción de los alumnos de ADE es en general más bajo que el de los de CC. EE., en general la satisfacción mostrada por los alumnos (ADE + CC. EE.) es considerable para con todos aquellos aspectos relacionados con la organización académica (siempre superior a 3,2), el proceso de enseñanza-aprendizaje (siempre superior a 3,3), los sistemas de evaluación del alumno (superior a 3,4) y la atención al estudiante, si bien en este último apartado tanto los alumnos de ADE como los de cc. EE. asignan un nivel de satisfacción bajo $(2,8)$ al horario de atención al estudiante.

Si la satisfacción general con todos los aspectos académicos es positiva, no ocurre lo mismo con el nivel de satisfacción de los alumnos con las instalaciones y servicios, ya que de los 13 aspectos tratados tan sólo 4 de ellos obtienen una media de satisfacción igual o superior a 3 .

TABla 1. EVAluación Del PROCESO InSTITUCiOnAL POR PARTE DE LOS ALUMNOS

\begin{tabular}{|c|c|c|c|c|}
\hline CRITERIO A EVALUAR & $\begin{array}{l}\text { MEDIA } \\
\text { ADE }\end{array}$ & $\begin{array}{l}\text { DESV. } \\
\text { ADE }\end{array}$ & $\begin{array}{l}\text { MEDIA } \\
\text { CC. EE. }\end{array}$ & $\begin{array}{l}\text { DESV. } \\
\text { CC. EE. }\end{array}$ \\
\hline \multicolumn{5}{|l|}{ ORGANIZACIÓN ACADÉMICA } \\
\hline El horario de la asignatura lo considero adecuado & 3,15 & 1,08 & 3,58 & 0,98 \\
\hline Conozco la información necesaria para matricularme & 3,55 & 0,92 & 3,56 & 0,92 \\
\hline $\begin{array}{l}\text { He podido obtener la información académica } \\
\text { que necesitaba antes de matricularme }\end{array}$ & 3,58 & 0,90 & 3,58 & 0,94 \\
\hline Se han producido cambios de horarios & 3,18 & 1,26 & 3,04 & 1,25 \\
\hline Las incidencias se han resuelto adecuadamente & 3,54 & 0,31 & 3,60 & 0,85 \\
\hline La coordinación entre teoría y práctica es adecuada & 3,56 & 0,89 & 3,65 & 0,91 \\
\hline $\begin{array}{l}\text { La coordinación entre profesores de } \\
\text { diferentes asignaturas es adecuada }\end{array}$ & 3,71 & 0,85 & 3,71 & 0,9 \\
\hline \multicolumn{5}{|l|}{ PROCESO ENSEÑANZA-APRENDIZAJE } \\
\hline $\begin{array}{l}\text { El conjunto de las asignaturas de este } \\
\text { año me parece adecuado }\end{array}$ & 3,56 & 0,96 & 3,67 & 0,92 \\
\hline $\begin{array}{l}\text { En este curso me han facilitado los temarios o } \\
\text { programas de las asignaturas al inicio del mismo }\end{array}$ & 3,65 & 1,01 & 3,62 & 0,95 \\
\hline $\begin{array}{l}\text { Los programas contienen información } \\
\text { útil, amplia y detallada }\end{array}$ & 3,60 & 0,80 & 3,67 & 0,70 \\
\hline Los profesores cumplen con los programas & 3,70 & 0,69 & 3,73 & 0,73 \\
\hline $\begin{array}{l}\text { El nivel de conocimientos impartidos se adecua } \\
\text { al perfil académico de los estudiantes }\end{array}$ & 3,45 & 0,76 & 3,42 & 0,69 \\
\hline Las clases prácticas se adecuan a la realidad laboral & 3,33 & 0,89 & 3,51 & 0,88 \\
\hline
\end{tabular}


TERESA FAYOS GARDÓ, MARTINA GONZÁLEZ-GALLARZA GRANIZO Y DAVID SERVERA FRANCÉS

PERCEPCIONES DE LOS DIFERENTES AGENTES IMPLICADOS EN EL SERVICIO DE FORMACIÓN UNIVERSITARIA

\begin{tabular}{|c|c|c|c|c|}
\hline $\begin{array}{l}\text { La metodología de los profesores es adecuada } \\
\text { al nivel y características de los estudiantes }\end{array}$ & 3,45 & 0,83 & 3,62 & 0,73 \\
\hline $\begin{array}{l}\text { Los materiales de estudio recomendados se } \\
\text { adaptan a las exigencias de los programas }\end{array}$ & 3,29 & 0,99 & 3,16 & 1,10 \\
\hline \multicolumn{5}{|l|}{ ATENCIÓN AL ESTUDIANTE } \\
\hline $\begin{array}{l}\text { Acudo habitualmente a los horarios } \\
\text { de atención al estudiante }\end{array}$ & 2,81 & 1,07 & 2,82 & 0,9 \\
\hline El horario de atención al cliente es viable para mí & 2,99 & 1,06 & 3,22 & 0,96 \\
\hline $\begin{array}{l}\text { Los profesores cumplen con su horario } \\
\text { de atención al estudiante }\end{array}$ & 3,62 & 0,93 & 3,55 & 0,86 \\
\hline $\begin{array}{l}\text { La atención que recibo en el horario } \\
\text { de tutorías es adecuada }\end{array}$ & 3,64 & 0,94 & 3,67 & 1 \\
\hline $\begin{array}{l}\text { La comunicación con los profesores es fluida y } \\
\text { adecuada, tanto en el aula como fuera de ella }\end{array}$ & 3,87 & 0,73 & 3,96 & 0,61 \\
\hline \multicolumn{5}{|l|}{ EVALUACIÓN } \\
\hline El nivel exigido se ajustaba a los contenidos impartidos & 3,56 & 0,94 & 3,47 & 0,77 \\
\hline Los instrumentos y criterios de evaluación son adecuados & 3,36 & 0,97 & 3,25 & 0,87 \\
\hline \multicolumn{5}{|l|}{\begin{tabular}{|l|l} 
INSTALACIONES Y SERVICIOS \\
\end{tabular}} \\
\hline Conserjería & 3 & 1,03 & 3,15 & 1,04 \\
\hline Secretaría & 3,26 & 1,09 & 3,15 & 1,15 \\
\hline Servicio de Reprografía & 2,44 & 1,33 & 2,80 & 1,24 \\
\hline Aulas & 2,91 & 1,13 & 2,80 & 1,24 \\
\hline Aulas informática & 2,64 & 1,15 & 2,84 & 1,15 \\
\hline Espacios de trabajo en común & 2,46 & 1,1 & 2,56 & 1,15 \\
\hline Biblioteca & 2,38 & 1,21 & 2,36 & 1,28 \\
\hline Cafetería & 2,5 & 1,29 & 2,65 & 1,38 \\
\hline Zonas deportivas & 2,56 & 1,24 & 2,75 & 1,31 \\
\hline Orientación al alumnado & 3 & 1,04 & 3,2 & 1,06 \\
\hline Servicio de comunicación & 3,05 & 0,95 & 3,16 & 1 \\
\hline Gabinete Médico & 2,69 & 1 & 2,91 & 1,01 \\
\hline Extensión universitaria & 2,77 & 1,09 & 2,89 & 1,03 \\
\hline
\end{tabular}

$(N A D E=62 ; N C C . E E .=55)$

Los siguientes resultados (Tabla 2) que complementan a los anteriores se recabaron de las consultas realizadas para la preparación de los nuevos grados. En este caso, el nivel de satisfacción mostrado por los alumnos confirma los anteriores bajos resultados obtenidos para los recursos y servicios en infraestructuras y equipamientos $(2,6)$, ya que estos vuelven a obtener la puntuación más baja en cuanto al grado de satisfacción de los alumnos de ADE y CC. EE. Tampoco es muy elevada la dimensión internacional del centro $(2,9)$, lo que sin duda es un resultado comprometido para la consecución de los objetivos del proceso de Bolonia. Conviene no obstante resaltar el alto índice de satisfacción que muestran los alumnos con la comunicación con el profesorado $(3,97)$. 


\section{2}

TERESA FAYOS GARDÓ, MARTINA GONZÁLEZ-GALLARZA GRANIZO Y DAVID SERVERA FRANCÉS

PERCEPCIONES DE LOS DIFERENTES AGENTES IMPLICADOS EN EL SERVICIO DE FORMACIÓN UNIVERSITARIA

En cuanto al grupo de delegados, también valoran como poco cualificados las instalaciones y servicios, mientras que asignan una buena valoración al proceso de enseñanza y aprendizaje. Los delegados subrayan también determinados aspectos que consideramos interesantes cara al desarrollo de los nuevos grados: los delegados requieren un programa de acción tutorial adaptado a las necesidades de cada uno de los cursos. Como es sabido, en primer curso, este es un aspecto que se ha implementado en forma de créditos básicos en algunos de los grados, con materias relativas a la incorporación a la universidad. Será interesante valorar la satisfacción futura del alumnado con esta nueva actividad propia de la implementación de los grados en España. También es interesante comentar que los canales de comunicación universidad-alumno más conocidos son los de la página weB y la Intranet, siendo también los que más se utilizan y valoran; y, por último, consideran que la atención que se recibe en el horario de tutorías es adecuada, no obstante, subrayan que no acuden habitualmente a los horarios de atención al estudiante.

Además de preguntar a los alumnos sobre su satisfacción, en esta ocasión se les preguntó sobre el grado de importancia que presentaban en su formación las distintas asignaturas cursadas o por cursar, obteniéndose unos resultados que conviene en primer lugar detallar y en segundo comparar con la opinión de los responsables de las empresas empleadoras, pronunciándose sobre la importancia otorgada a dichos conocimientos.

Así, tanto los alumnos de ADE como de CC. EE. consideran que las asignaturas que mayor nivel de importancia tienen en su formación son la informática y el inglés (aunque los alumnos de CC. EE. en menor medida esta última), seguidas a continuación de asignaturas relacionadas con la contabilidad, la tributación de la empresa y las finanzas empresariales. También los alumnos coinciden al apreciar que las asignaturas que consideran menos importantes en su formación (media de satisfacción inferior a 3) son todas aquellas relacionadas con las humanidades (ética, hombre y empresa, historia); este último resultado es muy contextual, al ser el centro investigado un centro privado. Estos resultados parecen ir en la línea de lo que debería ser el nuevo escenario de cambio en el diseño de los planes de estudio, un escenario en el que se priman las habilidades frente a los conocimientos.

TABla 2. Consultas Comisión GRAdo (ADE + CC. Ee.)

\begin{tabular}{|l|c|c|}
\hline \multicolumn{1}{|c|}{ CRITERIOS } & MEDIA & DESVIACIÓN \\
\hline Plan de estudios & 3,49 & 0,69 \\
\hline Desarrollo de las enseñanzas (planificación, metodología) & 3,58 & 0,62 \\
\hline Estudiantes (política de admisión, apoyo a los estudiantes) & 3,51 & 0,96 \\
\hline Personal docente & 3,52 & 0,75 \\
\hline Recursos y servicios (infraestructura y equipamiento) & 2,60 & 1,08 \\
\hline Dimensión internacional (movilidad de estudiantes) & 2,95 & 0,95 \\
\hline Resultados (tasa de éxito, tiempo invertido) & 3,72 & 0,70 \\
\hline
\end{tabular}


TERESA FAYOS GARDÓ, MARTINA GONZÁLEZ-GALLARZA GRANIZO Y DAVID SERVERA FRANCÉS

PERCEPCIONES DE LOS DIFERENTES AGENTES IMPLICADOS EN EL SERVICIO DE FORMACIÓN UNIVERSITARIA

\begin{tabular}{|c|c|c|}
\hline Comunicación profesores & 3,97 & 0,78 \\
\hline Recompensa (se reconocen y valoran los esfuerzos) & 3,68 & 0,87 \\
\hline Medios materiales (se gestionan de manera óptima) & 3,32 & 0,86 \\
\hline Compromiso personal de los directivos del centro & 3,33 & 0,80 \\
\hline Estructura organizativa & 3,25 & 0,84 \\
\hline Relación directivos-estudiantes & 3,38 & 0,85 \\
\hline Plan de acción tutorial & 3,41 & 1,08 \\
\hline
\end{tabular}

$(N=72)$

TABla 3. VALORACiÓN DE LAS ASIGNATURAS POR PARTE DE LOS ALUMNOS DE ADE

\begin{tabular}{|c|c|c|}
\hline ASIGNATURA & MEDIA & DESVIACIÓN \\
\hline Comportamiento del consumidor & 3,43 & 1,01 \\
\hline Contabilidad Financiera II & 4,10 & 0,77 \\
\hline Contabilidad General y Analítica & 4,15 & 0,83 \\
\hline Crecimiento empresarial & 3,24 & 0,69 \\
\hline Dirección comercial & 3,65 & 0,73 \\
\hline Dirección de RR. HH. & 3,38 & 0,94 \\
\hline Dirección estratégica y política de la empresa & 3,56 & 1,08 \\
\hline Dirección financiera & 4,37 & 0,69 \\
\hline Dirección y sucesión en la Empresa Familiar & 3,53 & 1,04 \\
\hline Econometría & 3,17 & 1,12 \\
\hline Economía Mundial y de España & 3,20 & 0,91 \\
\hline Estadística I & 3,47 & 0,89 \\
\hline Ética de la actividad empresarial & 2,67 & 1,10 \\
\hline Fundamentos de marketing & 3,34 & 0,96 \\
\hline Gestión de la calidad total & 2,89 & 0,99 \\
\hline Gestión de proyectos & 3,14 & 1,21 \\
\hline Gestión del comercio exterior & 3,59 & 1,07 \\
\hline Historia Económica mundial & 2,65 & 1,08 \\
\hline Hombre y empresa & 2,17 & 1,02 \\
\hline Informática & 4,39 & 0,89 \\
\hline Inglés de los Negocios & 4,47 & 0,71 \\
\hline Instrumentos financieros & 4,13 & 0,99 \\
\hline Investigación operativa aplicada & 3,59 & 0,96 \\
\hline Investigación y planificación comercial & 3,50 & 0,72 \\
\hline Marco jurídico de la actividad empresarial & 3,67 & 0,86 \\
\hline Marketing relacional y fidelización de clientes & 3,74 & 0,74 \\
\hline Matemáticas & 3,63 & 0,84 \\
\hline Matemáticas Financieras (MOF) & 4,10 & 0,95 \\
\hline Mercado de la Unión Europea & 3,36 & 0,91 \\
\hline Microeconomía II & 3,58 & 0,92 \\
\hline Negociación con entidades financieras & 3,97 & 0,98 \\
\hline
\end{tabular}


164 TERESA FAYOS GARDÓ, MARTINA GONZÁLEZ-GALLARZA GRANIZO Y DAVID SERVERA FRANCÉS PERCEPCIONES DE LOS DIFERENTES AGENTES IMPLICADOS EN EL SERVICIO DE FORMACIÓN UNIVERSITARIA

\begin{tabular}{|l|c|c|}
\hline Planificación financiera & 3,77 & 1,12 \\
\hline Procesos de auditoría y asesoría & 3,60 & 0,99 \\
\hline Promoción, comunicación e imagen & 2,95 & 1,00 \\
\hline Relaciones empresa y sociedad & 2,58 & 0,91 \\
\hline Sistema Financiero Español & 3,33 & 0,88 \\
\hline Sistemas de coste y de control de gestión & 3,54 & 0,94 \\
\hline Sistemas de pensamiento empresarial & 2,45 & 1,06 \\
\hline Sistemas informatizados de org. y adm. & 3,47 & 1,08 \\
\hline Tributación de la empresa & 4,02 & 0,79 \\
\hline
\end{tabular}

$(N=49)$

TABla 4. VALORACiÓN DE las ASignATURAS POR PARTE DE LOS ALUMNOS DE CC. EE.

\begin{tabular}{|l|c|c|}
\hline \multicolumn{1}{|c|}{ ASIGNATURA } & MEDIA & DESVIACIÓN \\
\hline Análisis Económico Financiero & 3,75 & 0,61 \\
\hline Contabilidad Financiera & 4,25 & 0,74 \\
\hline Dirección Comercial & 3,48 & 1,12 \\
\hline Dirección Financiera & 3,88 & 0,90 \\
\hline Economía española y mundial & 3,65 & 0,88 \\
\hline Economía política & 3,61 & 0,84 \\
\hline Estadística & 3,38 & 1,10 \\
\hline Ética de la Actividad Empresarial & 2,23 & 1,23 \\
\hline Fiscalidad de la Empresa I & 3,67 & 1,13 \\
\hline Gestión Calidad Total & 3,36 & 1,08 \\
\hline Gestión Comercio Exterior & 3,5 & 1,03 \\
\hline Gestión de Ventas & 3,75 & 1,00 \\
\hline Informática I & 4,09 & 0,51 \\
\hline Inglés de los Negocios I & 3,88 & 0,90 \\
\hline Introducción Régimen Jurídico de la Empresa & 3,65 & 0,71 \\
\hline Investigación Planificación Comercial & 3,59 & 0,87 \\
\hline Marketing y Ventas en el Comercio Exterior & 3,69 & 0,95 \\
\hline Matemáticas Empresariales & 3,73 & 0,83 \\
\hline Mercado Unión Europea & 3,47 & 1,07 \\
\hline MOF & 3,7 & 0,88 \\
\hline Negociación Entidades Financieras & 3,76 & 0,89 \\
\hline Organización y Administración de Empresas & 3,78 & 0,74 \\
\hline Planificación Financiera & 3,69 & 0,87 \\
\hline Práctica de Nóminas y Seguridad Social & 3,84 & 1,01 \\
\hline Producción, Distribución y Logística & 3,6 & 1,06 \\
\hline Régimen Jurídico de la Empresa & 3,6 & 0,99 \\
\hline Sistemas informatizados de Org. y Admón. & 3,47 & 0,87 \\
\hline N & & \\
\hline
\end{tabular}

$(N=24)$ 


\subsection{La perspectiva del empresariado}

Con objetivo de recabar la opinión de las empresas actualmente empleadoras y también futuras empleadoras de los alumnos de ADE y CC. EE. se les preguntó por el grado de importancia de las habilidades y conocimientos de los alumnos en el momento de su inserción en la empresa, obteniéndose los siguientes resultados.

En cuanto a las habilidades de los alumnos, las empresas asignan un muy elevado grado de importancia para todas ellas (tanto capacidades como habilidades), ya que la media asignada es siempre superior a 3,7, y para siete de ellas superior a 4. Destacan por su mayor importancia la capacidad para trabajar en equipo, la iniciativa, y la capacidad para resolver problemas y la capacidad de organización y planificación. No obstante, en relación con estas últimas capacidades, el diseño de las guías docentes que se propone en el desarrollo de los nuevos grados por la adaptación a Bolonia, consideramos que va en contra del fomento de dicha capacidad debido, entre otras razones, a la poca flexibilidad de los cronogramas y exhaustividad en las indicaciones que se deben cumplir.

A pesar de que todas ellas son consideradas importantes, las habilidades con menor grado de importancia asignadas son la habilidad para gestionar proyectos, la habilidad para la reflexión ética (coincidiendo por tanto en esta ocasión el alumnado y el empresariado) y el liderazgo (lo de manera cualitativa fue justificado por los alumnos ya que a un recién licenciado no se le suelen valorar este tipo de habilidades). No obstante, este aspecto, debería ser revisado ya que estas habilidades, que según la literatura sí son valoradas en otros sistemas educativos como el norteamericano, son en algún aspecto, como el fomento de la iniciativa y la gestión de proyectos, fundamentales para la formación de futuros empresarios.

Si las empresas asignaban valores altos de importancia a todas las habilidades y capacidades, no sucede lo mismo cuando se les pregunta sobre la importancia que tienen los conocimientos aprendidos por los alumnos, ya que de los 8 grupos de conocimientos sobre los que se les preguntó, sólo dos de ellos obtuvieron una media de importancia superior a 4 (a pesar de que todos la obtienen superior a 3). De estos dos grupos de conocimientos sorprende que además de obtener exactamente la misma media de importancia (4,23), ninguno está directamente relacionado con los contenidos de la titulación sino con conocimientos transversales, ya que son la informática y el inglés. Recordemos que de nuevo se produce una coincidencia con la importancia que los alumnos asignaban en su formación a estos conocimientos, ya que la informática y el inglés eran las asignaturas que los alumnos, especialmente los de ADE, consideraban más importantes.

En cuanto a los conocimientos relacionados directamente con la titulación los considerados más importantes por las empresas son los que dan lugar a una especialización del alumnado, es decir, las finanzas y el marketing. Así, los conocimientos del alumnado en el momento de su inserción empresarial considerados menos importantes por parte de las empresas son los conocimientos sobre gestión de los recursos humanos y sobre fiscalidad. 
166 TERESA FAYOS GARDÓ, MARTINA GONZÁLEZ-GALLARZA GRANIZO Y DAVID SERVERA FRANCÉS PERCEPCIONES DE LOS DIFERENTES AGENTES IMPLICADOS EN EL SERVICIO DE FORMACIÓN UNIVERSITARIA

TABLA 5. OPINIÓN DE LAS EMPRESAS

\begin{tabular}{|l|c|c|}
\hline \multicolumn{1}{|c|}{ HABILIDADES } & MEDIA & DESVIACIÓN \\
\hline Cultura básica empresarial & 3,78 & 10,82 \\
\hline Capacidad de razonar en términos económicos & 3,87 & 1,68 \\
\hline Capacidad para la resolución de problemas & 4,28 & 1,63 \\
\hline Capacidad de organización y planificación & 4,30 & 1,29 \\
\hline Capacidad de análisis y síntesis & 4,19 & 1,17 \\
\hline Identificación de problemas & 4,21 & 0,81 \\
\hline Habilidad para la Gestión de Proyectos & 3,67 & 0,88 \\
\hline Habilidad para analizar y buscar información & 4,02 & 0,89 \\
\hline Habilidad para la reflexión ética & 3,74 & 0,91 \\
\hline Habilidades de Comunicación & 4,09 & 0,89 \\
\hline Aprendizaje y desarrollo personal & 4,19 & 0,87 \\
\hline Capacidad de tomar decisiones & 4,04 & 0,88 \\
\hline Trabajo en equipo & 4,59 & 0,83 \\
\hline Iniciativa & 4,34 & 0,82 \\
\hline Liderazgo & 3,74 & 0,79 \\
\hline & MEDIA & DESVIACIÓN \\
\hline Informática & 4,23 & 0,77 \\
\hline Recursos Humanos & 3,11 & 0,90 \\
\hline Dirección Estratégica & 3,43 & 0,97 \\
\hline Contabilidad & 3,55 & 0,99 \\
\hline Finanzas & 3,72 & 0,99 \\
\hline Marketing/Comercial & 3,68 & 1,02 \\
\hline Fiscalidad & 3,30 & 1,04 \\
\hline Idiomas & 4,23 & 1,05 \\
\hline
\end{tabular}

$(N=48)$

\subsection{La perspectiva de los egresados}

Otro colectivo de interés son los propios egresados, que valoran el output de su formación en su inserción laboral y en su capacidad profesional. A pesar del bajo nivel de respuesta de este colectivo, conviene que destaquemos diferentes resultados que, aunque no concluyentes, pueden ser testados en futuras investigaciones con mayores muestras.

En primer lugar, subrayamos el elevado nivel de satisfacción de los egresados con los conocimientos adquiridos y su utilidad en su desempeño profesional, siendo su nivel de satisfacción inferior en cuanto a las habilidades desarrolladas. En segundo lugar, la proximidad y la profesionalidad de los profesores es altamente valorada por los egresados (recordamos que se trata de un centro privado de pequeño tamaño con una elevada relación profesor-alumno); en tercer lugar, los 
egresados consideran que las prácticas en empresa los preparan para integrarse en el mercado laboral, y, por último, trabajar en equipo es una de las habilidades que consideran que más han desarrollado durante su estancia en la Universidad, recordemos que esta era la habilidad considerada la más importante por el colectivo de empresarios.

\subsection{La perspectiva del profesorado}

De los resultados de las encuestas realizadas al profesorado, destacamos tres ideas importantes. La primera es la opinión expresada por este colectivo de que los estudiantes llegan a las universidades con manifiesta falta de interés y motivación. Este aspecto pone en cuestión algunos de los postulados de la necesaria orientación al alumno en los nuevos grados, basados en una madurez e independencia del alumno universitario, que no siempre es real. La segunda es que existe una brecha importante entre el estudiante real y el estudiante ideal. Las futuras valoraciones de las implantaciones de los grados, en sus acreditaciones y en las opiniones que reflejen los propios implicados, los estudiantes, podremos concluir sobre esta dicotomía que, por ahora, es una impresión del profesorado consultado. Y la tercera, más relacionada con la organización de la enseñanza, es que las exposiciones metodológicas y teóricas son utilizadas en mucha mayor medida que el trabajo individual e independiente del estudiante. Todas estas cuestiones son recogidas y apuntadas como retos para las autoridades responsables del diseño de titulaciones y, junto a las ya mencionadas anteriormente, se comentan en forma de conclusiones generales de este trabajo en un último epígrafe.

\section{CONCLUSIONES E IMPLICACIONES PARA LA GESTIÓN ACADÉMICA}

Buscando progresar en dos ejes de la particularidad del servicio universitario (su multidimensionalidad y la multiplicidad de agentes implicados, a los que añadimos, por ser explícitamente objetivo del proceso de Bolonia, el interés por la empleabilidad), este trabajo ha buscado explorar las opiniones y evaluaciones de diferentes agentes implicados en el servicio universitario: alumnos, egresados, empleadores y profesores. La lectura de los datos se ha hecho a la luz de sus posibles implicaciones para la nueva gestión estratégica del servicio universitario. Así, además de la consabida importancia de gestionar el servicio universitario como un servicio holístico, es decir, de carácter multidimensional, pero percibido como una agregabilidad, donde tan importantes son los servicios académicos como los paraservicios que lo rodean, este trabajo ha permitido desvelar lo que podríamos entender como algunas sombras de las implicaciones de los postulados de la Declaración de Bolonia a las particularidades del estudiante universitario actual.

Por un lado, el cambio anunciado de paradigma en el que la unidad de medida del trabajo son las horas dedicadas por el estudiante y no la estimación del 
profesor del tiempo dedicado en el aula a la exposición de cada tema hace suponer que el estudiante es un agente maduro y autónomo, capaz de organizar su propio aprendizaje; no obstante, la realidad social que vivimos en la Universidad en la última década es bien diferente; ¿está el estudiante que actualmente accede a la Universidad en condiciones de madurez para calibrar el trabajo personal y exigirse en autonomía y en esfuerzo lo que este nuevo enfoque propone? En esta investigación se ha constatado la opinión manifestada por los profesores relativa a la falta de interés y motivación de los estudiantes, lo que presenta un problema cara a la implantación de unas titulaciones basadas en la iniciativa y creatividad de trabajo del estudiante. Sin embargo, también la organización de la enseñanza tiene que dar un giro copernicano, en la que también los profesores individualmente y en colaboración con sus equipos directivos universitarios faciliten dicha iniciativa por parte de los estudiantes. Adicionalmente, estas habilidades son especialmente valiosas a los ojos de los empleadores, lo que confiere a este tema una proyección e importancia mayor. Este futuro alumno "maduro y autónomo" deberá poseer también, cara a su futura inserción profesional, capacidad para realmente trabajar en equipo y capacidad de comunicación. Las valoraciones de este aspecto deberán ser recogidas en las acreditaciones de los grados, en años venideros.

Por otro lado, una conclusión importante se deriva del resultado obtenido de que los conocimientos son considerados menos importantes que las capacidades y habilidades por parte de las empresas, y los conocimientos transversales o complementarios (informática e inglés, esencialmente) los más importantes. En este aspecto, coinciden los colectivos de empleadores, egresados y estudiantes, lo que le confiere por ello mayor importancia, y debe hacernos reflexionar a la hora de diseñar nuevas titulaciones. Este resultado sí está en consonancia con el planteamiento de los nuevos grados, sin embargo, se corre el riesgo de dejar de lado conocimientos de base importantes que a medio plazo disminuyan la capacidad de abstracción del alumno y su capacidad de desarrollar capacidades que contribuyan a la sinergia y cooperación con otros interlocutores, lo cual es imprescindible para el desarrollo de la innovación. El difícil equilibrio entre el "qué" y el "cómo" enseñar es uno de los mayores retos a los que se enfrenta el proceso de Bolonia en las titulaciones de empresa en España, en donde ya no existe una diferencia entre "diplomados" y licenciados", y en donde la reducción de las titulaciones en duración (en un 20\% al pasar de 5 a 4 años) no debería suponer una merma en los contenidos, incorporando necesariamente una aproximación más aplicada de la que se carecía.

Una cuestión adicional que permite ampliar el debate sobre los nuevos escenarios a los que nos enfrentamos en la mejor provisión del Servicio de Formación Universitaria es de naturaleza ya clásica pero que permite nuevos matices: nos referimos a la necesidad endógena de coordinación que conlleva la gestión académica, y que ahora se complica aun más con la inclusión de nuevos agentes implicados como son los empleadores (que cobran ahora mayor valor no sólo en la valoración del éxito del proceso, sino también en su planificación y diseño) o 
los padres, cada vez más presentes en la toma de decisiones no sólo en la universidad privada como pagadores, sino también en la universidad pública como prescriptores de sus hijos. En lo que respecta a los estudiantes, la valoración de su propia iniciativa en el diseño y adaptación de su propio currículo académico es un aspecto que se desprende de los resultados de este trabajo, pero que creemos que queda en entredicho, debido a la escasa flexibilidad de los cronogramas y exhaustividad en las indicaciones que se deben cumplir para la adaptación de las titulaciones anteriores. Este aspecto necesitará ser revisado en las futuras acreditaciones de los grados.

Como todo estudio empírico, este trabajo conlleva una serie de limitaciones, que reconocemos a continuación. El centro elegido para el trabajo de campo es un centro privado de tamaño reducido (con una elevada relación alumno-profesor) por lo que algunos de los colectivos estudiados (profesores, en primer lugar, y alumnos, en segundo) muestran una implicación mayor; los resultados deben por tanto extrapolarse con cautela. Metodológicamente, además, las diferentes muestras son todas de conveniencia, sin haberse por ello respetado estratificaciones por sexo, edad, curso, especialidad... El enfoque del trabajo ha sido más en amplitud que en profundidad, puesto que los resultados, aunque aplicados a 6 colectivos, son meramente descriptivos (medias) y no se realizan análisis bivariados que pudieran arrojar mayor luz sobre diferencias significativas entre colectivos, lo que dejamos para futuros trabajos. Estas reflexiones deben servir como orientaciones para futuras investigaciones o simplemente para alumbrar el seguimiento de la aplicación de estos nuevos retos a la realidad social y personal del estudiante universitario, tanto en España como en el resto de Europa.

\section{REFERENCIAS BIBLIOGRÁFICAS}

Angulo, F.; Pergelova, A. y Rialp, J. (2010) A market segmentation approach for higher education based on rational and emotional factors. Journal of Marketing for Higher Education, 20 (1), 1-17.

Appleton-Knapp, S. L. y Krentler, K. A. (2006) Measuring Student Expectations and Their Effects on Satisfaction: The importance of Managing Student Expectations. Journal of Marketing Education, 28, 254-264.

ARAmbeWela, R.; Hall, J. y ZuHair, S. (2005) Postgraduate International Students from Asia: Factors Influencing Satisfaction. Journal of Marketing for Higher Education, 15 (2), 105-128.

ATHIYAman, A. (1997) Linking student satisfaction and service quality perceptions: the case of university education. European Journal of Marketing, 31 (7/8), 528-541.

BeErli, A. y Díaz, G. (2003) Los efectos de la imagen percibida de la universidad en la satisfacción de los estudiantes. Revista Española de Investigación de Marketing, EsIC, 7 (1), 7-25.

BEKKERING, E. y ENGELland, B. (2002) Extending quality assessment beyond the classroom: the campus computer lab scale. Journal of Advancement of Marketing Education, 2, $18-24$ 
170 TERESA FAYOS GARDÓ, MARTINA GONZÁLEZ-GALLARZA GRANIZO Y DAVID SERVERA FRANCÉS PERCEPCIONES DE LOS DIFERENTES AGENTES IMPLICADOS EN EL SERVICIO DE FORMACIÓN UNIVERSITARIA

Belanger, C.; Mount, J. y Wilson, M. (2002) Institutional Image and Retention. Tertiary Education and Management, 8 (3), 217-230.

Boone, C.; Van Olfen, W. y Roijakkers, N. (2004) Selection on the Road to a Career: Evidence of Personality Sorting in Educational Choice. Journal of Career Development, 31 (1), 61-78.

Borrador Protocolo de Evaluación para la Verificación de Títulos Universitarios Oficiales. ANECA (15-11-2007). España.

BuRKE, R. (1997) Correlates of Under-employment among recent business school graduates. International Journal of Manpower, 18, 627-633.

Ceular, N.; Caridad, J. M.; NúÑez, J. y Roldán, J. A. (2008) Estudio de la evolución de los egresados de la universidad y su inserción en el panorama empresarial. Actas del 27 Congreso Internacional CIRIEC sobre economía pública, social y cooperativa. Sevilla (España).

Comm, C. L. y LabaY, D. G. (1996) Repositioning colleges using changing student quality perceptions: An exploratory analysis. Journal of Marketing for Higher Education, 7 (4), 21-35.

CONSEJO DE COORDINACIÓN UNIVERSITARIA DEL MEC (2003) La integración del sistema universitario español en el Espacio Europeo de Enseñanza Superior. Documento Marco. España.

CONSEJO EUROPEO (2004) Instrucción y formación 2010: la urgencia de las reformas para el impulso de la estrategia de Lisboa, 3-3-2004. Documento del Consejo 6905/2004.

Consejo General de Colegios de EConomistas de España (2008) Los economistas ante el Espacio Europeo de Educación Superior: análisis desde la experiencia y determinación de las necesidades futuras. Madrid, febrero de 2008.

CORRALES, E. y ROdRíguez, B. (2003) La transición del sistema educativo al mercado laboral. Análisis de los factores determinantes del primer empleo. Actas de las V Jornadas de Economía de la Educación. Universidad Rovira i Virgili.

Cuestas, P. J.; Fenollar, P. y Román, S. (2006) Las motivaciones sociales como antecedentes del éxito académico de los estudiantes universitarios de marketing. Ponencia presentada en los XVIII Encuentros de Profesores Universitarios de Marketing. Almería, 20, 21 y 22 de septiembre de 2006.

De Miguel, M. (dir.); Alfaro, I. J.; Apodaca, P.; Arias, J. M.; García, E. y Pérez, A. (2005) Adaptación de los planes de estudio al proceso de convergencia europea. Oviedo: Servicio de publicaciones de la Universidad de Oviedo.

ENGELland, B. T.; WORKMAN, L. y Sing, M. (2000) Ensuring service quality for campus career services centers: a modified SERVQUAL scale. Journal of Marketing Education, 22 (3), $236-45$.

Fielder, J. S.; Hilton, C. B. y Motes, W. H. (1993) Educational services marketing: a proposed system for enhanced recruitment of students. Journal of Professional Services Marketing, 8, 191-205.

FilippaKOU, O. y TAPPER, T. (2008) Quality Assurance and Quality Enhancement in Higher Education: Contested Territories? Higher Education Quarterly, 62 (1/2), 84-100.

Fuentes, M. y Gil, I. (2006) Segmenting University Students on the basis of their expectations. Journal of Marketing for Higher Education, 16 (1), 25-45.

Fuentes, M.; Gil, I. y BerEnguer, G. (2005) Las expectativas de los estudiantes universitarios. Una aproximación como base de segmentación. Ponencia presentada en XVII Encuentros de Profesores Universitarios de Marketing. Madrid, septiembre de 2005. 
Fuentes, M.; Gil, I. y Moliner, B. (2007) La utilidad de las expectativas como base de segmentación predictiva de la calidad del servicio universitario, en VI Congreso Internacional de Marketing Público y No lucrativo. Braga 14-15 de junio de 2007.

Gallarza, M. G.; Fayos, T.; Servera, D. y ArTeaga, F. (2008) Análisis y evaluación del Servicio de Formación Universitaria: implicaciones para el marketing estratégico de las universidades. 7th International Congress Marketing Trends. ESCP-EAP VENECIA, enero 2008.

Grady, N. B.; Fisher, D. L. y Fraser, B. J. (1996) Images of school through metaphor development and validation of a questionnaire. Journal of Educational Administration, 34 (2), 41-53.

Hemsley-Brown, J. y Oplatka, I. (2006) Universities in a competitive global marketplace. A systematic review of the literature on higher education marketing. International Journal of Public Sector Management, 19 (4), 316-338.

Informe ANECA al Consejo de Universidades sobre los procesos de evaluación derivados de la LOU (2008). 15 de julio de 2008. ANECA.

IVY, J. (2001) Higher education institution image: a correspondence analysis approach. International Journal of Educational Management, 15 (6), 276-282.

KrampF, R. F. y Heinlein, A. C. (1981) Developing marketing strategies and tactics in higher education through target market research. Decision Sciences, 12 (2), 175-93.

Leblanc, G. y NGUYEN, N. (1999) Listening to the customer's voice: examining perceived service value among businesses college students. International Journal of Educational Management, 13 (4), 187-198.

Ley Orgánica de modificación de la LOU: Ley Orgánica 4/2007, de 12 de abril, por la que se modifica la Ley Orgánica 6/2001, de 21 de diciembre. (LOU). BOE. España.

Luque, T. y Del Barrio, S. (2007) Análisis del valor de las percepciones de los clientes en el diagnóstico estratégico de la universidad. Ponencia presentada en el International Congress Marketing Trends, 26-27 de enero de 2007.

MANSFIELD, P. M. y WARWICK, J. (2005) Gender differences in Students' and Parents' Evaluative Criteria When Selecting a College. Journal of Marketing for Higher Education, 15 (2), 47-80.

MANZANEQue, M. y BARBA, V. (2008) Experiencia de planificación docente adaptada a los criterios del EEES para la licenciatura de administración y dirección de empresas en la Facultad de Ciencias Sociales de Cuenca. Aportaciones para la implantación del nuevo título de grado. VI Jornadas de Redes de Investigación en Docencia Universitaria. Alicante, junio 2008.

MARINGE, F. (2006) University \& Course Choice: Implications for positioning, recruitment and marketing. International Journal of Educational Management, 20 (6), 466-479.

Neave, G. y Amaral, A. (2008) On Process, Progress, Success and Methodology or the Unfolding of the Bologna Process as it Appears to Two Reasonably Benign Observers. Higher Education Quarterly, 62 (1/2), 40-62.

NGuYen, N. y Leblanc, G. (2001) Image and reputation of higher education institutions in student's retention decisions. International Journal of Educational Management, 15 (6), 303-311.

NÚÑEZ, E. (2007a) Marketing ocupacional: Análisis de las actitudes de los jóvenes ante la formación profesional y el trabajo. Ponencia presentada en el XIX Encuentro de Profesores Universitarios de Marketing (EPUM), septiembre de 2007.

- (2007b) Marketing ocupacional: La transición de la educación al mercado laboral en los estudiantes de formación profesional de la Comunidad Autónoma de Madrid. Ponencia 
172 TERESA FAYOS GARDÓ, MARTINA GONZÁLEZ-GALLARZA GRANIZO Y DAVID SERVERA FRANCÉS PERCEPCIONES DE LOS DIFERENTES AGENTES IMPLICADOS EN EL SERVICIO DE FORMACIÓN UNIVERSITARIA

presentada en el XIX Encuentro de Profesores Universitarios de Marketing (EPUM), septiembre 2007.

Oosterbeek, H.; Groot, W. y Hartog, J. (1992) An Empirical Analysis of University Choice and Earnings. The Economist, 140 (3), 293-309.

Pampaloni, A. M. (2010) The influence of organizational image on college selection: what students seek in institutions of higher education. Journal of Marketing for Higher Education, 20 (1), 19-48.

PaRAMESWARAN, R. y GlowaCKa, A. E. (1995) University image: an information processing perspective. Journal of Marketing for Higher Education, 6 (2).

R. D. 1044/2003, de 1 de agosto, por el que se establece el procedimiento para la expedición por las universidades del suplemento europeo al título. BOE. España.

R. D. $1467 / 2007$, de 2 de noviembre, por el que se establece la estructura del bachillerato y se fijan sus enseñanzas mínimas. BOE. España.

R. D. $1125 / 2003$, de 5 de septiembre: Sistema europeo de créditos y sistema de calificaciones. Definición ECTS. BOE. España.

R. D. 1993/2007 de 29 de octubre: Ordenación de las enseñanzas universitarias oficiales. $B O E$. España.

RAHONA, M. (2004) ¿La posesión de un título universitario facilita el acceso de los jóvenes al primer empleo? Una aproximación para el caso español. Revista del Ministerio de Trabajo y Asuntos Sociales, 61, 105-119.

- (2006) La influencia del entorno socioeconómico en la realización de estudios universitarios: una aproximación al caso español de la década de los 90. Hacienda Pública Española/Revista de Economía Pública, 178 (3), 55-80.

Ribera-CAmino, J. y De JuAn, M. D. (2007) Educación de calidad en Marketing: un modelo holístico. Ponencia presentada en el XIX Encuentro de Profesores Universitarios de Marketing (EPUM), septiembre 2007.

Servera, D.; González-Gallarza, M.; Fayos, T. y ArTeaga, F. (2008) La evaluación del Servicio de Formación Universitaria: análisis de las opiniones de los diferentes agentes implicados. VI Jornadas de Redes de Investigación en Docencia Universitaria. Alicante, junio 2008.

Smith, D. y ADAms, J. (2008) Academics or Executives? Continuity and Change in the Roles of Pro-vice-chancellors. Higher Education Quarterly, 62 (4), 340-357.

SOUTAR, G. N. y TURNER, J. P. (2002) Students' preferences for university: a conjoint analysis. International Journal of Educational Management, 16 (1), 40-45.

Tchibozo, G. (2007) Extra-Curricular Activity and the Transition from Higher Education to Work: A Survey of Graduates in the United Kingdom. Higher Education Quarterly, 61 (1), 37-56.

UniversitÀ Degli STUdi Di Bologna. The European Space for Higher Education (1999) The European Higher Education Area. Joint declaration of the European Ministers of Education. Convened in Bologna on the $18^{\text {th }}$ of June, 1999.

VANDER SCHEE, B. A. (2010) The small college enrollment officer: relationship marketing at work. Journal of Marketing for Higher Education, 20 (1), 135-143.

VIDAL, J. (coord.) (2003) Métodos de análisis de la inserción laboral de los universitarios. Ministerio de Educación y Cultura.

Whitchurch, C. (2008) Shifting Identities and Blurring Boundaries: the Emergence of Third Space Professionals in UK Higher Education. Higher Education Quarterly, 62 (4), 377-396.

ZABALZA BERAZA, M. Á. (2004) Guía para la planificación didáctica universitaria en el marco de la EEES. Universidad de Santiago de Compostela. Octubre. 